\title{
Algebraic Structures in local QFT
}

\author{
D. Kreimer ${ }^{\mathrm{a} *}$ \\ ${ }^{a}$ Institut des Hautes Etudes Scientifiques, 35 rte. de Chartres, \\ 91440 Bures sur Yvette, France
}

\section{Acknowledgments}

It is a pleasure to thank the participants of the Les Houches school on Structures in local quantum field theory 1, Les Houches June 07-25 2010, where many of the results summarized here were presented in detail, for a great and stimulating three weeks. And similarly, thanks to the Loops and Legs community [2], for the constant stimulus which nurtures any investigation into the inner workings of quantum fields.

\section{The polylog as a Hodge structure}

It is the purpose of this short review to emphasize the (limiting) mixed Hodge structures underlying quantum field theory $3 \sqrt[415]{3}$. We do so by comparison with the well understood structure of the polylogarithm $[\underline{6}$.

We emphasize that the latter has a representation as an iterated integral, and hence also an underlying Hopf algebra structure, see [7. Indeed, consider the three columns $\left(C_{1}, C_{2}, C 3\right)$ which form a matrix

$$
\left(\begin{array}{ccc}
1 & 0 & 0 \\
-\mathrm{Li}_{1}(z) & 2 \pi i & 0 \\
-\mathrm{Li}_{2}(z) & 2 \pi i \ln (z) & (2 \pi i)^{2}
\end{array}\right) .
$$

This Hodge structure, apparent in the interplay of colums which compensate their variations amongst each other (here, a mathematicians variations are a physicists discontinuities along branch cuts) allows the definition of an invariant

$\operatorname{Var}\left(\Im L i_{2}(z)-\ln |z| \Im L i_{1}(z)\right)=0$.

The Hodge sructure and the Hopf algebra structure cooperate: If we define $L\left(t_{k}\right)=\mathrm{L}_{k}(z) \equiv$

\footnotetext{
*Work supported in parts by grant NSF/DMS-0603781 at
} Boston University. $\ln ^{k} z / k !, \operatorname{Li}\left(t_{k}\right)=\operatorname{Li}(z)$ with Hopf algebra structure the free commutative algebra on generators $t_{k}$ with coproduct $\Delta\left(t_{k}\right)=\sum_{j=0}^{k} t_{j} \otimes t_{k-j}$, then the columnwise branch cut ambiguities compensate in a combination familiar to a physicist from Bogoliubov's R-operation [7]:

$$
\operatorname{Var}\left(m\left(L^{-1} \otimes L i \circ P\right) \Delta\left(t_{k}\right)\right)=0 .
$$

Moreover, Griffith transversality leads to a differential equation which uniquely defines the dilogarithm from $\mathrm{L}_{1}(z)$ and $\operatorname{Li}_{1}(z)$, and similar for the higher polylogs.

Let us now return to Feynman graphs.

\section{Hopf algebra of graphs}

This is a well-studied subject by now [8], so we just list the main formulae. The Hopf algebra is a free commutative algebra, graded by the loop number: $H=\mathbb{Q} 1 \oplus \bigoplus_{j=1}^{\infty} H^{j}=\mathbb{Q} 1+P H$ with a projector $P$ into the augmentation ideal. It hence furnishes:

i) the coproduct:

$$
\Delta(\Gamma)=\Gamma \otimes 1+1 \otimes \Gamma+\overbrace{\sum_{\gamma=\cup_{i} \gamma_{i}, \omega_{4}\left(\gamma_{i}\right) \geq 0} \gamma \otimes \Gamma / \gamma}^{\Delta^{\prime}(\Gamma)}(3)
$$

ii) the antipode:

$S(\Gamma)=-\Gamma-\sum S(\gamma) \Gamma / \gamma=-m(S \otimes \mathrm{P}) \Delta$

iii) the character group:

$G_{V}^{H} \ni \Phi \Leftrightarrow \Phi: H \rightarrow V, \Phi\left(h_{1} h_{2}\right)=\Phi\left(h_{1}\right) \Phi\left(h_{2}\right)(5)$

iv) the counterterm (parametrized by a RotaBaxter map $R$ ):

$S_{R}^{\Phi}(\Gamma)=-R\left(\Phi(h)-\sum S_{R}^{\Phi}(\gamma) \Phi(\Gamma / \gamma)\right)$ 


$$
=\quad-R \Phi\left(m\left(S_{R}^{\Phi} \otimes \Phi P\right) \Delta(\Gamma)\right)
$$

v) the renormalized Feynman rules:

$\Phi_{R}=m\left(S_{R}^{\Phi} \otimes \Phi\right) \Delta=[\mathrm{id}-R] m\left(S_{R}^{\Phi} \otimes \phi P\right) \Delta$.

\section{An Example}

The co-product $\left(\Delta^{\prime}\right.$ indicates projection into $P H$ on both sides):

$$
\begin{aligned}
& \Delta^{\prime}(\Delta \otimes)=3 \otimes \\
& +2 m_{3} \otimes+\cdots \otimes \text {. }
\end{aligned}
$$

The counterterm:

$$
\begin{aligned}
& S_{R}^{\Phi}(x)= \\
& -R m\left[S_{R}^{\Phi} \otimes \Phi P\right] \times \\
& \times \Delta(\Delta, \Phi) \\
& =-R\{\Phi(\Phi)+ \\
& +R[\Phi(3+2 \quad+\cdots)] \Phi(\curvearrowright)\}
\end{aligned}
$$

The renormalized result:

$$
\begin{array}{r}
\Phi_{R}=(\mathrm{id}-R) m\left(S_{R}^{\Phi} \otimes \Phi P\right) \times \\
\times \Delta( \\
=(\mathrm{id}-R)\{\Phi( \\
+R[\Phi(3 \\
+2
\end{array}
$$

\section{4. sub-Hopf algebras}

As a commutative graded Hopf algebra, $H$ has a Hochschild cohomology [9. This leads to the study of sub-Hopf algebras arising from linear combinations of generators contributing at a given order. We let $r$ indicate an amplitude from the set $\mathcal{R}$ needing renormalization. Summing order by order:

$$
\begin{gathered}
c_{k}^{r}=\sum_{|\Gamma|=k, \operatorname{res}(\Gamma)=r} \frac{1}{|\operatorname{Aut}(\Gamma)|} \Gamma \\
\Rightarrow \Delta\left(c_{k}^{r}\right)=\sum_{j} \operatorname{Pol}_{j}\left(c_{m}^{s}\right) \otimes c_{k-j}^{r} .
\end{gathered}
$$

The relevant polynomials $\mathrm{Pol}_{j}$ can be easily obtained, see 101112 for example.

Hochschild closedness says $b B_{+}^{r ; j}=0$. Hence,

$\Delta B_{+}^{r ; j}(X)=B_{+}^{r ; j}(X) \otimes 1+\left(i d \otimes B_{+}^{r ; j}\right) \Delta(X)$.
It reduces the sum over all graphs to a sum over primitives (which does not improve the asymptotics that much after all [13])

$$
X^{r}=1 \pm \sum_{j} c_{j}^{r} \alpha^{j}=1 \pm \sum_{j} \alpha^{j} B_{+}^{r ; j}\left(X^{r} Q^{j}(\alpha)\right),
$$

$Q^{j}=\frac{X^{v}}{\sqrt{\prod_{\text {edges e at v }} X^{e}}}$.

The latter evaluates to the Lorentz invariant charge under the renormalized Feynman rules. This set-up implies locality of counterterms upon application of Feynman rules $\Phi B_{+}^{r ; j}(X)=$ $\int d \mu_{r ; j} \Phi(X)$ :

$$
\left.\bar{R}(\Gamma)=m\left(S_{\Phi}^{R} \otimes \Phi P\right)\right) \Delta B_{+}^{r ; j}=\int d \mu_{r ; j} \Phi_{R}(X),
$$

from the co-radical filtration and Hochschild cohomology of $H$.

\section{Symmetry}

The study of such sub-Hopf algebras is significant to understand internal symmetry. Ward and Slavnov-Taylor identities appear naturally in this context as co-ideals. Combinations of graphs as

$i_{k}:=c_{k}^{\bar{\psi} \psi}+c_{k}^{\bar{\psi} A \psi}$

span Hopf (co-)ideals $I$ :

$\Delta(I) \subseteq H \otimes I+I \otimes H$.

For example, in QED to two loops,

$$
\begin{aligned}
\Delta\left(i_{2}\right)= & i_{2} \otimes 1+1 \otimes i_{2}+\left(c_{1}^{\frac{1}{4} F^{2}}+c_{1}^{\bar{\psi} A \psi}+i_{1}\right) \\
& \otimes i_{1}+i_{1} \otimes c_{1}^{\bar{\psi} A \psi} .
\end{aligned}
$$

Feynman rules vanish on $I: \Phi_{L}^{R}(\{\Theta\})(\iota)=0, \forall \iota \in$ $I \Leftrightarrow$ Feynman rules respect quantized symmetry: $\Phi_{R}: H / I \rightarrow V$.

Note that this vanishing on $I$ implies the classical equation of motion for the tree-level terms which we obtain from shrinking internal edges. Ideals for Slavnov-Taylor identities are generated by equality of renormalized charges [10[14]. Indeed. the whole set-up for the master equation in Batalin-Vilkovisky goes through (see Walter van 
Suijlekom's work [12]). For generalizations of this set-up to Hopf algebras treating all 1PI subgraphs as sub-objects, see [11]. This has in particular bearing on the the BCFW recursion and quantum gravity, which leave much to explore for the future.

\section{Kinematics and Cohomology}

The above Hochschild cohomology will not only generate the correct perturbation expansion, symmetry factors included, but has consequences in understanding scattering, upon understanding the role of exact one-cocycles.

Exact co-cycles $b \phi^{r ; j}$, one for each closed cocycle $B_{+}^{r ; j}$, are images of the Hochschild coboundary operator $b, b \circ b=0$, of maps $\phi^{r} ; j: H \rightarrow \mathbb{C}$. The equivalence class is hence described as

$\left[B_{+}^{r, j}\right]=B_{+}^{r ; j}+b \phi^{r ; j}={\tilde{B_{+}}}^{r ; j}$.

It has its natural role in describing the variation of scattering angles in renormalized amplitudes, using dedicated such $\phi^{r ; j}: H \rightarrow \mathbb{C}$ for each generator of the cohomology class. Indeed, we look at the variation of external momenta separated as a variation of an overall scale and angles defined by that scale:

$G_{R}^{r}(\{g\}, \ln s,\{\Theta\})=1 \pm \Phi_{R}^{\ln s,\{\Theta\}}\left(X^{r}(\{g\})\right)$

with $X^{r}=1 \pm \sum_{j} g^{j} B_{+}^{r ; j}\left(X^{r} Q^{j}(g)\right), b B_{+}^{r ; j}=0$, and

$G_{R}^{r}=\left[\sum_{j=1}^{\infty} \gamma_{j}^{r}(\{g\},\{\Theta\}) \ln ^{j} s\right]+\overbrace{G_{0}^{r}}^{\text {abelian factor }}$

Here, the abelian factor summarizes all terms which contribute to overall convergent formfactors in the Green function. They all can be subsumed by extending the group $\operatorname{Spec}(H)$ by an abelian factor, corresponding to the cocommutative and commutative Hopf algebra of convergent graphs.

Then, for MOM and similar schemes (not MS!):

$$
\{\Theta\} \rightarrow\left\{\Theta^{\prime}\right\} \Leftrightarrow B_{+}^{r ; j} \rightarrow B_{+}^{r, j}+b \phi^{r, j},
$$

describes the variation of angles at a fixed scale $L$ through the addition of an exact term, while

$\Phi_{L_{1}+L_{2},\{\Theta\}}^{R}=\Phi_{L_{1},\{\Theta\}}^{R} \star \Phi_{L_{2},\{\Theta\}}^{R}$. generates 1-parameter groups of automorphism coming from rescaling at fixed angles.

\section{Leading log expansions and the RGE}

For each vertex $v$, we have a combinatorial charge $Q^{v}$ :

$Q^{v}(g)=\frac{X^{v}(g)}{\prod_{e} \sqrt{X^{e}}}$

$e$ adjacent to $v$. Under the renormalized Feynman rules $\Phi_{R}, Q^{v}$ evaluates to a Poincaré invariant charge of the theory (it is not invariant under conformal transformations - charges run when dilated). Using the underlying Hopf algebra structure, the renormalization group equation (RGE)

$$
\left(\partial_{L}+\beta(g) \partial_{g}-\sum_{e a d j r} \gamma_{1}^{e}\right) G^{r}(g, L)=0
$$

rewrites in terms of the Dynkin operator $\left(\gamma_{1}^{r}(g)=\right.$ $\left.S \star Y\left(X^{r}(g)\right)\right):$

$\gamma_{k}^{r}(g)=\frac{1}{k}\left(\gamma_{1}^{r}(g)-\sum_{j \in R} s_{j} \gamma_{1}^{j} g \partial_{g}\right) \gamma_{k-1}^{r}(g)$,

as the expected recursion for the leading log expansion [16 17].

\section{Ordinary differential equations vs DSE}

The series over all graphs $X^{r}$ contributing to an amplitude $r$ was given above as a solution to a fixpoint equation in Hochschild cohomology. Under $\Phi_{R}$, this fixpoint equation turns into the familiar Dyson-Schwinger equations (DSE), and the distinguished primitive elements $B_{+}^{r ; j}(1)$ turn into the skeleton integral kernels for these equations. It is a remarkable fact that the resulting sub-Hopf algebras are invariant on any finite truncation of the sum over skeletons [15]. Using the iterated integral structure (see [18] for a formal exposition of iterated integrals)

$\Phi_{R}\left(B_{+}^{r ; j}(X)\right)=\int \Phi_{R}(X) d \mu_{r ; j}$

allows to combine $X^{r}=1 \pm \sum_{j} B_{+}\left(X^{r} Q^{j}\right)$ with the RGE to a system of ordinary differential equa- 
tions $\forall r \in \mathcal{R}$

$\gamma_{1}^{r}=P(g)-\left[\gamma_{1}^{r}(g)\right]^{2}+\sum_{j \in \mathcal{R}} s_{j} \gamma_{1}^{j} g \partial_{g} \gamma_{1}^{r}(g)$,

for the linear term in the leading log expansion, whilst the higher terms are determined as before, see (19).

We have a single equation for the $\beta$-function in massless gauge theories: $\beta(g)=g \gamma_{1}(g) / 2$, for $\gamma_{1}$ the anomalous dimension of the massless gauge propagator

$\gamma_{1}(g)=P(g)-\gamma_{1}(g)\left(1-g \partial_{g}\right) \gamma_{1}(g)$.

This uses the Ward identity in QED and a background field gauge for QCD, see below.

A few words concerning the function $P$, whose existence we assume, are appropriate. It contains certainly the periods $\operatorname{res}(p)$ which are generated by primitive $1 \mathrm{PI}$ graphs $p \in H$ as coefficient of the term linear in $L, \Phi_{R}(p)=\operatorname{res}(p) L$. What else? To understand this, consider two primitives $p_{1}, p_{2}$, and assume there are $n_{1}$ insertion places for $p_{2}$ in $p_{1}$, and $n_{2}$ for $p_{1}$ in $p_{2}$.

Define $n_{+}=\left(n_{1}+n_{2}\right) / 2, n_{-}=\left(n_{1}-n_{2}\right) / 2$. Then, using the pre-Lie insertion of graphs $\star$, the symmetric

$n_{+}\left(\frac{1}{n_{1}} p_{1} \star p_{2}+\frac{1}{n_{2}} p_{2} \star p_{2}-p_{1} p_{2}\right)$,

is a primitive element in the Hopf algebra generated by a suitable Dynkin operator, whilst the antisymmetric

$n_{-}\left(\frac{1}{n_{1}} p_{1} \star p_{2}-\frac{1}{n_{2}} p_{2} \star p_{2}\right)$

also evaluates to only a term linear in $L$, and can best be interpreted as the dual of the normalized commutator

$\left[Z_{p_{1}}, Z_{p_{2}}\right] \in L$

in the Lie algebra $L$ dual to $H$. The primitives $p$, and their symmetric extensions above, correspond all to elements in the center of the Lie algebra $L$ appearing in $H=U^{\star}(L)$ by Milnor Moore. We thus expect that a dedicated study of the lower central series of said Lie algebra is mandatory to understand $P$ fully, to incorporate the commutator subgroups correctly.

\section{Limiting mixed Hodge structures}

The Hopf algebra $H$ can be obtained from flags

$f:=\gamma_{1} \subset \gamma_{2} \subset \ldots \subset \Gamma, \Delta^{\prime}\left(\gamma_{i+1} / \gamma_{i}\right)=0$.

The set of all such flags $F_{\Gamma} \ni f$ determines the Hopf algebra structure. We let $\left|F_{\Gamma}\right|$ be the length of the flag, while the number of flags is determined from the decomposition into maximal forests, and hence is sensitive to the number of overlapping subdivergences [3].

The flag decomposition then determines a column vector $v=v\left(F_{\Gamma}\right)$ and a nilpotent matrix $(N)=\left(N\left(\left|F_{\Gamma}\right|\right)\right),(N)^{k+1}=0, k=\operatorname{corad}(\Gamma)$ such that

$$
\begin{array}{r}
\lim _{t \rightarrow 0}\left(e^{-\ln t(N)}\right) \Phi_{R}\left(v\left(F_{\Gamma}\right)\right)= \\
\left(c_{1}^{\Gamma}(\Theta) \ln s, c_{2}^{\Gamma}(\Theta), c_{k}^{\Gamma}(\Theta) \ln ^{k} s\right)^{T}
\end{array}
$$

where $k$ is determined from the co-radical filtration and $t$ is a regulator say for the lower boundary in the parametric representation.

The vector on the rhs is a vector of periods in the mathematical sense. Renormalization hence appears as a limiting mixed Hodge structure. In fact, 3] contains a full treatment for arbitrary internal masses and external momenta of that limit, leading to a renormalized integrand which is a well-defined projective differential form with a log-homogenous integrand. This opens renormalized amplitudes to the same study by algebrogeometric methods, as recently applied to the periods provided by primitive graphs.

The study of these periods actually initiated the discovery of the Hopf algebra structures underlying QFT here reported. It started in collaboration with David Broadhurst [19, and after the connection to algebraic geometry was established in 5, the study of these periods recently blossomed thanks to the works of Francis Brown 20 21], Dzmitry Doryn [25 26], Oliver Schnetz [22 23] and Karen Yeats [24]. The progress is mainly due to a detailed study of the linear algebra and algebraic geometry of the two Kirchhoff polynomials, which also leads to an improved understanding of Green's function as functions of internal masses and external momenta 44. 


\section{The Feynman graph as a Hodge struc- ture}

Let us dwell a moment on the last point. In general, to learn about the analytic structure of Feynman integrals as functions of external momenta and internal masses, we start from a Hopf algebra structure as above. Here we exhibit the simplest case of one-loop graphs, which provide the simplest primitives of $H$. Actually, we focus on the one-loop triangle:

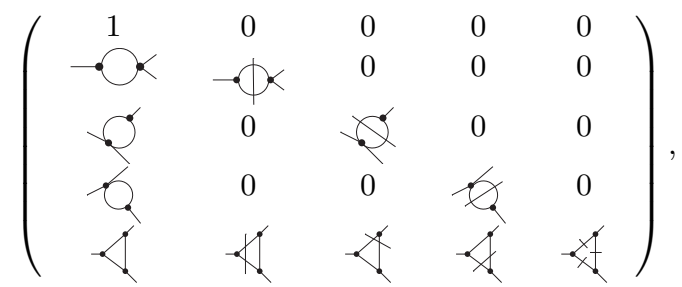

which provides five column vectors $\left(C_{1}, C_{2}, C_{3}, C_{4}, C_{5}\right)$, say. In the first column, the first entry 1 corresponds to the tree level term. Then, the next three entries are the three reduced diagrams which we can assign to the one-loop triangle graph. The fifth entry is finally the triangle itself. Columns 2,3,4 then put suitable internal propagators on the mass shell, hence capturing suitable variations of the entries in the first column.

Note that the two cuts in the reduced diagrams leave no free integration, but give a mass and momenta dependent constant which generalizes the entry $2 \pi i$ in the Hodge structure of the dilog in Eq.(11). The triangle cut at two internal edges leaves one free integration of the form $\int d z /(a z+b)$, corresponding to the entry $\ln z$ before.

Finally, the last column has three internal propagators on the mass shell. It hence exceeds what Cutkosky would tell us, and closes the above matrix to a Hodge matrix by the results of [4. The recent physicists practice to put more internal propagators on the mass shell than prescribed by Cutkosky has indeed, it seems, a nice mathematical origin, see 4. It is tempting to muse that many questions regarding cut-reconstructability could be clarified by clarifying the Hodge structure underlying quantum field theory.

So for the future: what, then, can we learn from invariant triangles as in

$$
\operatorname{Var}(\Im \cdot\rfloor-[\Re \cdot \lambda \cdot \Im \cdot \backslash]+\cdots)=0,
$$

considered as functions of complex external momenta and internal masses?

\section{QED}

We now turn to aspects of resummation of Feynman diagrams, and hence to rather modest first attempts to connect the Hodge and Hopf algebra approach exhibited here to nonperturbative physics. Lacking precise knowledge of the generalized skeleton function $P(x)$, we study what can be said as a function of assumptions on $P(x)$. We start with QED [27. It suffices to consider the sub Hopf algebra for vacuum polarization graphs. We are led to a single ODE:

$\gamma_{1}(x)=P(x)-\gamma_{1}(x)^{2}+\gamma_{1}(x) x \partial_{x} \gamma_{1}(x)$,

with $P(x)>0$ and $P(x)$ twice differentiable assumed. Also, at some small coupling $x_{0}$, we assume $\gamma_{1}\left(x_{0}\right)=\gamma_{0}>0$.

We then find different solutions distinguished by flat $e^{-\frac{1}{x}}$ behaviour but with an identical perturbation expansion near the origin. Switching to the coupled system with the running charge included, $\frac{d \gamma_{1}}{d x}=\gamma_{1}-\gamma_{1}^{2}-P, \frac{d x}{d L}=x \gamma_{1}, L=$ $\int_{x_{0}}^{x(L)} \frac{d z}{z \gamma_{1}(z)}$, we find three types of solutions with a separatrix separating those above which all have a Landau pole, from those below which are double valued and hence unphysical, see Fig.(1). The separatrix exists under rather mild conditions on $P(x)$ and might or might have not a Landau pole, as a function of much finer asymptotics of $P(x)$ for large coupling $x$. See Karen Yeats talks at [1], or see [27/28, for details.

\section{QCD}

Again, the sub Hopf algebra for gluon polarization graphs suffices, now upon using the background field gauge [28. We have the same ODE,

$\gamma_{1}(g)=P(g)-\gamma_{1}(g)^{2}+\gamma_{1}(g) g \partial_{g} \gamma_{1}(g)$,

with $P(g)<0$. We assume $P(g)$ twice differentiable and concave near 0 . Now, the separatrix is indeed clearly the solution preferred by 


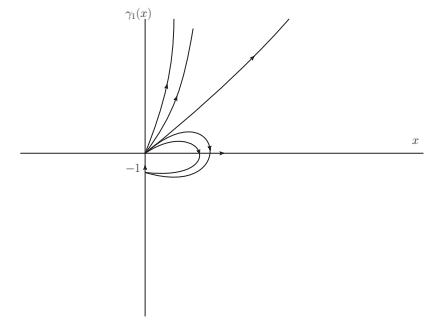

Figure 1. QED: The $\beta$-function $\left(\beta=\gamma_{1} / 2\right)$ as a function of the coupling. The separatrix is the only solution which might have no Landau pole.

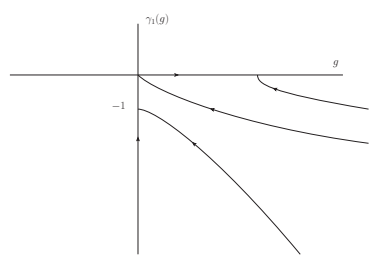

Figure 2. QCD: The $\beta$-function $\left(\beta=\gamma_{1} / 2\right)$ as a function of the coupling. Only the separatrix flows into the origin at high energy.

physics: its the unique solution which flows into $(0,0)$ at large $Q^{2}$. The scale of QCD is then set by $L=\int_{g_{0}}^{g(L)} \frac{d z}{z \gamma_{1}(z)} \rightarrow L_{\Lambda}=-\int_{g\left(L_{\Lambda}\right)}^{\infty} \frac{d z}{z \gamma_{1}(z)}$, $L_{\Lambda}=\ln Q^{2} / \Lambda_{Q C D}$.

For $P(g)<0$, the picture is given in Fig.(2). The separatrix exists and gives an asymptotic free solution, with a finite mass gap possibly for the inverse propagator iff $\gamma_{1}(x)<-1$ for some $x>0$. See 28. for technical details using for dispersive methods as introduced by Shirkov et.al. in field theory.

\section{REFERENCES}

1. See http://math.bu.edu/people/dkreimer/houches/houches.html.

2. See https://indico.desy.de/conferenceDisplay. py? conf Id=2200.

3. S. Bloch and D. Kreimer, Commun. Num. Theor. Phys. 2 (2008) 637.
4. S. Bloch, D. Kreimer, Feynman amplitudes and Landau singularities for 1-loop graphs, preprint, available via [1.

5. S. Bloch, H. Esnault and D. Kreimer, Commun. Math. Phys. 267 (2006) 181.

6. S. Bloch. In: Structural properties of polylogarithms (L. Lewin, ed.), 275285, Math. Surveys Monogr., 37, Amer. Math. Soc., Providence, RI, 1991.

7. D. Kreimer. In: Noncommutative Geometry and Number Theory. Edited by C. Consani and M. Marcolli. Vieweg, 2006. ppf. 187-204.

8. K. Ebrahimi-Fard and D. Kreimer, J. Phys. A 38 (2005) R385.

9. A. Connes and D. Kreimer, Commun. Math. Phys. 199 (1998) 203.

10. D. Kreimer, Annals Phys. 321 (2006) 2757.

11. D. Kreimer and W. D. van Suijlekom, Nucl. Phys. B 820 (2009) 682.

12. see Walter van Suijlekom's lectures in [1, and references there.

13. D. J. Broadhurst, Z. Phys. C 58 (1993) 339.

14. W. D. van Suijlekom, Commun. Math. Phys. 276 (2007) 773.

15. C. Bergbauer and D. Kreimer, IRMA Lect. Math. Theor. Phys. 10 (2006) 133.

16. D. Kreimer and K. Yeats, Nucl. Phys. Proc. Suppl. 160 (2006) 116.

17. Thesis. Boston University 2008. AMS, in print. arXiv:0810.2249.

18. F. Brown, Lectures ion iterated integrals, available via [1].

19. D. J. Broadhurst and D. Kreimer, Phys. Lett. B 393 (1997) 403.

20. F. Brown, arXiv:0910.0114.

21. F. Brown, Commun. Math. Phys. 287 (2009) 925.

22. O. Schnetz, arXiv:0801.2856.

23. F. Brown, O. Schnetz, arXiv:1006.4064,

24. F. Brown, K. Yeats, arXiv:0910.5429.

25. D. Doryn, arXiv:0811.0402 (2008).

26. D. Doryn, arXiv:1006.3533v1 (2010).

27. G. van Baalen, D. Kreimer, D. Uminsky and K. Yeats, Annals Phys. 324 (2009) 205.

28. G. van Baalen, D. Kreimer, D. Uminsky and K. Yeats, Annals Phys. 325 (2010) 300. 